UMDPP\#99-015

\title{
Defect Formation and Critical Dynamics in the Early Universe
}

\author{
G. J. Stephens ${ }^{*}$, E. A. Calzetta ${ }^{\dagger 2}$, B. L. Hu ${ }^{\ddagger 1}$, S. A. Ramsey ${ }^{\S} 1$ \\ ${ }^{1}$ Department of Physics, University of Maryland, College Park, Maryland 20742-4111 \\ ${ }^{2}$ Department of Physics and IAFE, University of Buenos Aires, Argentina
}

(August 9, 2018)

\begin{abstract}
We study the nonequilibrium dynamics leading to the formation of topological defects in a symmetry-breaking phase transition of a quantum scalar field with $\lambda \Phi^{4}$ self-interaction in a spatially flat, radiation-dominated FriedmannRobertson Walker Universe. The quantum field is initially in a finitetemperature symmetry-restored state and the phase transition develops as the Universe expands and cools. We present a first-principles, microscopic approach in which the nonperturbative, nonequilibrium dynamics of the quantum field is derived from the two-loop, two-particle-irreducible closed-timepath effective action. We numerically solve the dynamical equations for the two-point function and we identify signatures of topological defects in the infrared portion of the momentum-space power spectrum. We find that the density of topological defects formed after the phase transition scales as a power law with the expansion rate of the universe. We calculate the equilibrium critical exponents of the correlation length and relaxation time for this model and show that the power law exponent of the defect density, for both overdamped and underdamped evolution, is in good agreement with the "freeze-out" scenario proposed by Zurek. We also introduce an analytic dynamical model, valid near the critical point, that exhibits the same power-law scaling of the defect density with the quench rate. By incorporating the realistic quench of the expanding Universe our approach illuminates the dynamical mechanisms important for topological defect formation and provides a preliminary step towards a complete and rigorous picture of defect formation
\end{abstract}

*Electronic address: gstephen@physics.umd.edu

${ }^{\dagger}$ Electronic address: calzetta@df.uba.ar

$\ddagger$ Electronic address: hub@physics.umd.edu

$\S$ Electronic address: sramsey@physics.umd.edu 
in a second-order phase transition of a quantum field. The observed power law scaling of the defect density with the quench rate, calculated here in a quantum field theory context, provides evidence for the "freeze-out" scenario in three spatial dimensions.

PACS number(s): 11.27.+d, 98.80.Cq, 64.60.Ht, 05.70.Ln, 11.10.Wx 


\section{INTRODUCTION}

\section{A. Motivation}

Phase transitions in the early Universe played a decisive role in shaping the Universe we observe today. In the hot early Universe it is likely that the broken symmetries of the standard model of particle physics were partially or totally restored in a grand unified theory (GUT) and that a number of phase transitions occurred as the Universe expanded and cooled. These include transitions at the GUT scale $T_{c} \approx 10^{14}-10^{16} \mathrm{GeV}$, at the electroweak scale $T_{c} \approx 10^{2} \mathrm{GeV}$ and the color deconfinement and/or chiral phase transitions of QCD with $T_{c} \approx 100 \mathrm{MeV}$. An inflationary phase transition, a possible explanation for the flatness, horizon and monopole problems of the standard Friedmann-Robertson-Walker (FRW) cosmology, may have occurred at the GUT scale or before. At still earlier epochs near the Planck energy, $T_{c} \approx 10^{19} \mathrm{GeV}$, candidate theories of quantum gravity may allow for a phase transition to occur and give rise to the classical properties of spacetime described by general relativity. These first moments of the Universe contain clues to the solution of such outstanding problems as the origin of large-scale structure and matter-antimatter asymmetry and the nature of dark matter. The study of the consequences of cosmological phase transitions may therefore hold the key to our understanding of the Universe.

In a field theory with degenerate vacua, a symmetry-breaking phase transition can produce topological defects. Relics of the high-temperature symmetric phase of the theory, topological defects are topologically stable field configurations that are locally trapped in an excited state above the vacuum. Examples of defects include vortices in type II superconductors, superfluid Helium-3 and Helium-4, and cosmic strings formed in the early Universe. Topological defects are classified by the homotopy groups $\Pi_{n}(M)$ of the vacuum manifold, $M$. In three spatial dimensions simple defects are domain walls if $\Pi_{0}(M) \neq 1$, strings if $\Pi_{1}(M) \neq 1$, and monopoles if $\Pi_{2}(M) \neq 1$ [1].

Kibble was the first to show that topological defects are a generic feature of cosmological phase transitions [2]. In the course of a symmetry-breaking phase transition, as the field decays to the stable vacuum, it can choose the same vacuum state on length scales only as large as the correlation length. In the laboratory, if the phase transition proceeds slowly, the correlation length is bounded only by the size of the system and the field will effectively choose a homogeneous vacuum state. In the early Universe, however, the correlation length is bounded by the size of the particle horizon. With correlated regions limited in size by causality, Kibble argued that phase transitions in the early Universe necessarily leave a domain structure of vacuum states and form topological defects.

Topological defects formed in the early Universe can have profound consequences on the subsequent spacetime evolution. In the standard FRW cosmology, GUT scale monopoles, even if formed with an initial density equal to Kibble's lower bound of one defect per horizon volume, contribute more than $10^{12}$ times the largest possible density of the Universe consistent with observations. The overproduction of monopoles is a puzzle within the standard FRW cosmology and provided part of the original motivation for an early epoch of inflation [3]. Other topological defects such as cosmic strings are viable candidates for the seeds of structure formation and would produce a characteristic signature in the angular power spectrum of the cosmic microwave background. Satellites such as MAP and Plank designed 
specifically to measure fluctuations in the cosmic microwave background radiation to high accuracy are scheduled to be launched in the near future. The data obtained from these missions is expected to clarify the precise role of topological defects in the origin of structure formation in the Universe.

The importance of topological defects as candidates for the seeds of structure formation provides strong motivation to extract predictions from defect models that can be compared with observations. These predictions generally contain three main ingredients: the density of topological defects immediately following the completion of the phase transition, the subsequent evolution of the defect distribution and the calculation of physical observables such as cosmic microwave background radiation anisotropies and polarization. While both numerical and analytical work has been done on the evolution of the defect distribution and the extraction of physical predictions, less attention has been focused on quantitative predictions of the initial defect density which provides the starting conditions for the evolution of the defect distribution.

The dynamic origins of defect formation are also themselves of considerable physical interest. Formed in out-of-equilibrium processes, defects offer insight into the nature of critical dynamics. In addition, the ubiquitous nature of phase transitions links the study of the early Universe with the laboratory and allows the observation of cosmologically important mechanisms in condensed matter systems [4] [5]. Recently, researchers have exploited these similarities by observing defect formation in liquid crystals [6] and superfluid Helium [7]. In either condensed matter systems or the early Universe, the density of topological defects immediately following the phase transition is of substantial physical importance.

\section{B. The Kibble and Zurek Mechanisms of Defect Formation}

The first estimate of the initial defect density in a cosmological context was made by Kibble [2]. The basic ingredients of the Kibble mechanism are causality and the Ginzburg temperature, $T_{G}$. The Ginzburg temperature is defined as the temperature at which thermal fluctuations contain just enough energy for correlated regions of the field to overcome the potential energy barrier between inequivalent vacua,

$$
k_{b} T_{G} \sim \xi\left(T_{G}\right)^{3} \Delta F\left(T_{G}\right)
$$

where $\Delta F$ is the difference in free energy density between the true and false vacua and $\xi$ is the equilibrium correlation length. In the Kibble mechanism, the length scale characterizing the initial defect network is set by the equilibrium correlation length of the field, evaluated at $T_{G}$. In a recent series of experiments [7], the Kibble mechanism was tested in the laboratory. The results, while confirming the production of defects in a symmetry-breaking phase transition, indicate that $\xi\left(T_{G}\right)$ does not set the characteristic length scale of the initial defect distribution. These experiments were suggested by Zurek, who criticized Kibble's use of equilibrium arguments and the Ginzburg temperature, $T_{G}$ [4].

Combining equilibrium and non equilibrium ingredients, Zurek offers a "freeze-out" proposal to estimate the initial density of defects. In the Zurek proposal, above the critical temperature, the field starts off in thermal equilibrium with a heat bath. As the temperature of the bath is lowered adiabatically, the field remains in local thermal equilibrium 
with the heat bath. Near the phase transition, the equilibrium correlation length and the equilibrium relaxation time of the field grow without bound as

$$
\begin{gathered}
\xi=\xi_{0}|\epsilon|^{-\nu}, \\
\tau=\tau_{0}|\epsilon|^{-\mu}
\end{gathered}
$$

where $\epsilon$ characterizes the proximity to the critical temperature,

$$
\epsilon=\frac{T_{c}-T}{T_{c}}
$$

and $\mu$ and $\nu$ are critical exponents appropriate for the theory under consideration. The quench is assumed to occur linearly in time

$$
\epsilon=\frac{t}{t_{Q}}
$$

so that for $t<0$, the temperature of the heat bath is above the critical temperature and the critical temperature is reached at $t=0$. The divergence of the equilibrium relaxation time as the heat bath approaches the critical temperature is known as critical slowing down. Critical slowing down results from the finite speed of propagation of perturbations of the order parameter. As the correlation length diverges, small perturbations of the order parameter (e.g., lowering of the temperature) take longer to propagate over correlated regions and therefore it takes longer to reach equilibrium. As the critical temperature is approached from above there comes a time $|t *|$ during the quench when the time remaining before the transition equals the equilibrium relaxation time

$$
|t *|=\tau(t *)
$$

Beyond this point the correlation length can no longer adjust fast enough to follow the changing temperature of the bath. At time $t^{*}$ the dynamics of the correlation length "freezes". The correlation length remains frozen until a time $t^{*}$ after the critical temperature is reached. In Zurek's proposal the correlation length at the "freeze-out" time $t^{*}$ sets the characteristic length scale for the initial defect network. Solving for the value of the correlation length at $t^{*}$, the frozen correlation length, and therefore the initial defect density, scale with the quench rate as

$$
\xi(t *) \sim \tau_{Q}^{\frac{\nu}{1+\mu}}
$$

Aspects of this scenario have been verified using 1-dimensional and 2-dimensional simulations of phenomenological time-dependent Landau-Ginzburg equations [8] [9]. Preliminary experimental efforts to test the Zurek prediction in condensed matter systems are inconclusive, lacking reliable error estimates and a mechanism to vary the quench time scale [7].

The Kibble prediction for the initial length scale of the defect distribution is inconsistent with experiment, yet the alternative "freeze-out" proposal, raises many conceptual questions. The "freeze-out" scenario of Zurek relies on phenomenological ideas derived from experience 
with classical Landau-Ginzburg systems. It is not clear how much of this picture, if any, is applicable to quantum fields which undergo phase transitions in the early Universe. Quantum fields bring new conceptual issues. In particular, consideration of decoherence is necessary to understand when and how the quantum system at the late stages of the transition can be described by an ensemble of classical defect configurations. Even in the classical context, the use of equilibrium critical scaling in a fully dynamical setting is an approximation. Critical dynamics is generally much richer and less universal than equilibrium critical behavior [10]. For a system coupled to a rapidly changing environment, the correlation length evolves through the dynamical equations of motion of the field. Worse still, the relaxation time is not always well-defined. In addition, a realistic bath consists of interactions between the system and other fields in the Universe. Physical interactions between the bath and the system produce interesting and nonequilibrium behavior such as noise and dissipation [11]. The reduction of the complicated bath-system interaction to one parameter, the quench time scale, and a prescribed linear time-dependence in the effective mass of the time-dependent Landau Ginzburg equation neglects these potentially important processes.

\section{Foundational Issues}

Despite significant effort, the physical mechanisms important for the formation of topological defects in the early Universe and condensed matter systems are not well understood. It is useful to explore three interwoven and complementary themes that are relevant to this problem. These are (i) equilibrium vs. nonequilibrium, (ii) microscopic vs. macroscopic, and (iii) quantum vs. classical.

\section{Equilibrium vs. Dynamical}

The Zurek and Kibble mechanisms both rely on aspects of equilibrium physics. However, as emphasized by Zurek, the formation of topological defects is a nonequilibrium process. If aspects of the "freeze-out" scenario are correct then it must be viewed as an approximation to a more fundamental and as yet unexplored understanding. The initial density of topological defects formed in far-from-equilibrium phase transitions may be determined by very different mechanisms.

\section{Microscopic vs. Macroscopic}

Topological defects appear as configurations of the classical order parameter field in phenomenological Landau-Ginzburg theories and in the classical limit of symmetry-broken quantum field theories. A first-principles approach to the formation of topological defects in a nonequilibrium quantum field phase transition requires an understanding of the microscopic origin of macroscopic dynamical critical behavior like critical slowing down. Macroscopic domains of correlated vacuum must be identified from the microscopic quantum field. Ther-

malization occurs through microphysical couplings between the quantum field composing 
the system and other fields composing an environment and must be understood to produce a physical bath-system interaction.

\section{Quantum vs. Classical}

Phase transitions occur in quantum field theory and in condensed matter systems. In a second-order phase transition, the correlation length diverges at the critical temperature and the dynamics is dominated by low frequency modes. In equilibrium, modes with $\omega<<T$ have essentially a Maxwell-Boltzmann distribution and it might be expected that secondorder phase transitions are dominated by classical behavior. In a dynamical setting an exact equilibrium distribution does not exist and a necessary indication of classical behavior is the emergence of a positive definite probability distribution function from the density matrix of the quantum field [12]. In quantum critical systems decoherence may modify critical scaling exponents and change the coarsening of domains from that of classical systems. Even the concepts used to describe phase transitions in condensed matter may not apply in the quantum context. For example, the definition of a topological defect in a quantum field theory with arbitrary quantum state is unknown.

Phase transitions and topological defects occur in both classical and quantum systems, under equilibrium and nonequilibrium conditions and are depicted by phenomenological and microscopic theories. They therefore provide a theoretical vantage point from which to further elucidate the connections between these complementary and disparate concepts. The formation of topological defects is an arena in which these general yet basic issues of physics may be studied in a concrete and useful manner.

\section{Quantum Field Dynamics and the Formation of Topological Defects in the Early Universe}

A complete understanding of the physical issues involved in the formation of topological defects in a second-order phase transition in the early Universe requires a first-principle approach to the nonequilibrium dynamics of quantum fields, a realistic treatment of the interaction of the quantum field both with gravity and with other fields that constitute an environment, and the identification of classical defect configurations from the quantum field system.

This work will concentrate on the dynamics of the phase transition important for defect formation. While symmetry restoration in finite-temperature quantum field theory has been known since the early work of Kirzhnitz and Linde [13], most previous efforts have focused on the equilibrium aspects of the transition. Techniques such as the finite-temperature effective potential [14 [15] and the renormalization group [16] have been developed to deduce equilibrium critical properties such as the order of the phase transition and its critical temperature. However, equilibrium techniques are inadequate to study the dynamics of the phase transition. The use of equations of motion for the mean field derived from the finite-temperature effective potential was criticized in [17]. In general the use of equations generated from the finite-temperature effective potential in a dynamical setting results in 
unphysical solutions. Although equilibrium techniques are clearly inappropriate, solving the full equations of motion for an interacting field theory is generally impossible, even numerically. This difficulty is partially overcome by the development of approximation schemes which allow for the evolution of a restricted set of correlation functions of the quantum field theory [25] [26]. These methods have been applied to a number of dynamical problems in quantum field theory including post-inflationary reheating [18] (and references therein) and spinodal decomposition [19] [20] [12].

The problem of defect formation in a nonequilibrium second-order phase transition of a quantum field has also recently received attention [21] [22] [23]. The results are promising but the studies are incomplete. In these previous approaches the phase transition is incorporated through an ad hoc time dependence of the effective mass of a free field theory: an instability in the theory is induced when the mass becomes tachyonic. The use of a prescribed time dependence of the effective mass, while providing a convenient analytic model, lacks physical justification. The neglect of interactions confines the applicability of these approaches to very early times before the field amplitude grows substantially. They are therefore unable to account for the back reaction which is necessary to stabilize domain growth and shut off the spinodal instabilities of the phase transition. In addition, defects formed during the linear stages of the phase transition are transient and not likely to survive to late times.

\section{E. Outline}

In this paper we analyze the formation of correlated domains of true vacuum in the time evolution of a quantum scalar field during a second-order phase transition initiated by cooling of a radiation-dominated FRW Universe. In Section II we discuss the formalism used to follow the dynamics of a quantum field through the phase transition. We also present the model, a derivation of the equations of motion for the two-point function of the theory, and discuss renormalization, initial conditions and numerical parameters used in the numerical simulation. We then provide a dynamical description of the phase transition using results of the numerical simulations. Section III begins the discussion of domains and presents the argument that domains are determined by a peak in the Fourier space structure function $k^{2} G(k, t)$. Section IV discusses the power law scaling of the size of domains with the quench rate. The equilibrium critical exponents of the correlation length and relaxation time are calculated in both the underdamped and overdamped cases and the power law scaling of

the defect density with the quench rate predicted by the "freeze-out" proposal is shown to be in good agreement with the numerical simulations. An analytical model valid for slow quenches and near the onset of the instability is introduced and the power law exponent in the analytic model is found to be the same as the numerical simulations. Section V provides a summary and discussion of these results and presents possible directions for further study.

\section{PHASE TRANSITION DYNAMICS}

It is common to model critical dynamics with a classical, phenomenological, timedependent Landau-Ginzburg equation for an order parameter $\Psi$, 


$$
\partial_{t} \Psi(\vec{x}, t)=-\Gamma \frac{\delta F}{\delta \Psi}+\xi
$$

where $F[\Psi]$ is a phenomenological free energy density for the order parameter, $\Gamma$ is a phenomenological dissipative coefficient and $\xi$ is a stochastic term incorporating thermal fluctuations of the environment [24].

Even if the order parameter is of quantum origin, as e.g. in the phase of the wavefunction for liquid Helium-4, the Landau-Ginzburg equation is rarely derived logically from the underlying quantum dynamics of the system. In condensed matter systems, to compensate for insufficient microscopic information, great care with physical intuition goes into choosing the order parameter and its equation of motion. In experimentally inaccessible environments, such as the early Universe, it is not a priori obvious what the order parameter, or its dynamics, should be. In situations where phenomenological approaches are inadequate, it is necessary to work with the fundamental quantum dynamics of the fields.

A first-principles approach to the quantum dynamics of phase transitions avoids ad hoc assumptions about the dynamics of the correlation length and the effect of the quench. The system simply evolves under the true microscopic equations of motion. We can therefore explore many details of critical dynamics that are inaccessible in phenomenological theories.

Solving the exact dynamics of interacting quantum fields expressed, for example, through the Heisenberg equations of motion for the field operator is a very complicated problem. Instead, we seek a truncation of the full degrees of freedom that allows for an accurate modeling of the dynamics of the phase transition over the time scales of interest.

Consider a classical $\Phi^{4}$ scalar field theory with $m^{2}>0$ and self-coupling $\lambda$. The potential $V[\Phi]$ for the field is

$$
V[\Phi]=-\frac{1}{2} m^{2} \Phi^{2}+\frac{\lambda}{4} \Phi^{4}
$$

The degenerate true minima of this potential are

$$
\Phi_{\min }= \pm \frac{m}{\sqrt{\lambda}}
$$

which are nonperturbatively large in the coupling constant. To follow the field as it evolves from the unstable vacuum $\Phi=0$ to the stable one $\Phi= \pm \frac{m}{\lambda}$ requires an approach which is both non-perturbative in the field amplitude and fully dynamical.

A useful formalism that is both nonperturbative and dynamical is the two-particle irreducible closed-time-path (2PI-CTP) or in-in effective action [25] [27]. The 2PI-CTP effective action generates real and causal equations of motion for the mean field $\langle\Phi(\vec{x}, t)\rangle$ and twopoint correlation functions $\left\langle\Phi(\vec{x}, t) \Phi\left(\vec{y}, t^{\prime}\right)\right\rangle$ of the quantum field theory. A diagrammatic expansion of the 2PI effective action, to arbitrary loop order is given in [27]. Calculated to all orders in a loop expansion, the 2PI-CTP effective action and the equations of motion derived from it contain all information of the original quantum field theory. However, any practical computation requires inclusion of terms only to some finite loop order which constitutes an approximation [26] 28]. In this paper, we neglect three-loop and higher graphs. The truncation of the 2PI-CTP effective action to two-loop order is equivalent to the time dependent Hartree-Fock approximation [27]. 
The equations of motion for the mean field and the two-point function derived from the 2PI-CTP effective action respect the $\Phi \rightarrow-\Phi$ symmetry of the classical action. Since the field starts in a symmetry-restored state above the critical point where

$$
\langle\Phi\rangle_{\text {initial }}=0,
$$

the mean field remains identically zero throughout the phase transition. The dynamics of the phase transition unfolds through the dynamics of the two-point function.

The choice to limit attention to the two-point correlation functions in the Hartree-Fock approximation limits the time scale over which the evolution is physically reliable. Higher order correlations are suppressed in weakly coupled field theories in equilibrium. However, second-order phase transitions are characterized by spinodal instabilities that cause correlations to grow. When the correlations grow large enough that the field is sampling the stable vacua, dissipative processes are expected to become important and the Hartree-Fock approximation will break down [20].

\section{A. The Model}

We consider a scalar field in an FRW spacetime [29] [30]. The field has the symmetrybreaking classical action

$$
S=\int d^{4} x \sqrt{-g}\left(\partial_{\mu} \Phi \partial^{\mu} \Phi+m^{2} \Phi^{2}-\frac{\lambda}{4 !} \Phi^{4}\right)
$$

where $g$ is the determinant of the metric of the classical background spacetime. We assume that the stress-energy tensor is dominated by other radiation fields present in the early Universe. These fields maintain the overall homogeneity and isotropy of the Universe. Small deviations in homogeneity and isotropy that may eventually be responsible for the fluctuations observed in the cosmic microwave background radiation are produced in our model by the topological defects of the system and appear only at the end of the phase transition. We therefore work in the semiclassical test-field approximation (ignoring back reaction of the $\Phi$ field on the spacetime), and we assume that the scale factor has the time-dependence of a homogeneous and isotropic, spatially flat, radiation-dominated Universe,

$$
a(t)=\left[\frac{t+\tau}{\tau}\right]^{\frac{1}{2}} .
$$

The expansion of the Universe and the resulting redshifting of the modes act here as a physical quench allowing the dynamics of the phase transition to unfold naturally. This is in distinction to work which uses an instantaneous change in the sign of the square of the mass [20] [21].

In the Heisenberg representation the field operator $\Phi_{H}(\vec{x}, t)$ can be written as

$$
\Phi_{H}(\vec{x}, t)=\int d^{3} \vec{k}\left[e^{i \vec{k} \cdot \vec{x}} f_{k}(t) a_{\vec{k}}+e^{-i \vec{k} \cdot \vec{x}} f_{k}^{*}(t) a_{\vec{k}}^{\dagger}\right]
$$

where the $f_{k}$ are the complex quantum modes of the field. 
In a FRW Universe, the two-loop 2PI equation of motion in comoving cosmological time for the mode function with comoving momentum $\mathrm{k}$ is

$$
\left(\frac{d^{2}}{d t^{2}}+3 \frac{\dot{a}(t)}{a(t)} \frac{d}{d t}+\frac{k^{2}}{a^{2}(t)}-m^{2}+\frac{\lambda}{2} G(t, t)\right) f_{k}(t)=0,
$$

where

$$
G(t, t)=\int \frac{d^{3} k}{(2 \pi)^{3}} f_{k}(t) f_{k}^{*}(t) \sigma_{k}(\beta)
$$

is the equal-time limit of the two-point correlation function and

$$
\sigma_{k}(\beta)=\operatorname{coth}\left(\frac{\beta}{2} w_{k}(0)\right)
$$

is a constant factor incorporating thermal initial conditions with temperature $T=1 / \beta$. The effective mass of the system is

$$
m_{e f f}^{2}(t)=-m^{2}+\frac{\lambda}{2} G(t, t)
$$

and the initial frequency is

$$
w_{k}^{2}(0)=k^{2}+m_{e f f}^{2}(0)
$$

where $m_{\text {eff }}^{2}(0)$ is the initial finite-temperature effective mass in the Hartree-Fock approximation. Since we work in a radiation-dominated FRW universe, the scalar curvature, $R$, is zero and the conformal coupling constant $\xi$ may be ignored.

\section{Renormalization}

The equal-time limit of the two-point function is divergent and must be regularized. A simple regularization method, amenable to a numerical simulation, is to implement an ultraviolet cutoff in physical spatial momentum. A suitably regularized expression for the two-point function must be independent of this cutoff. Our renormalization scheme follows that of [29] 30].

It is a general feature of quantum field theory that the bare quantities appearing in the classical Lagrangian are not observable but are "dressed" by interactions. In the Hartree approximation, the effect of interactions is encoded in the self-consistent effective mass. Therefore, there are no counterterms to absorb divergences and the equation for the mode functions, Eq. (2.8), must be finite [31]. We therefore fix the renormalization scheme with the condition

$$
-m_{B}^{2}+\frac{\lambda_{B}}{2} G_{B}(t, t)=-m_{R}^{2}+\frac{\lambda_{R}}{2} G_{S}(t, t) .
$$

The cutoff dependence of the bare variance is obtained by considering a WKB-type solution to the mode function equation. Identifying the second order adiabatic mode functions from the WKB solution of the mode function equation, the subtracted two-point function is 


$$
G_{S}(t, t)=\frac{1}{2 \pi^{2}} \int_{0}^{\Lambda} k^{2} d k\left(f_{k} f_{k}^{*} \sigma_{k}(\beta)-\frac{1}{k a^{2}(t)}+\frac{\theta(k-\kappa)}{4 k^{3}} m_{e f f}^{2}\right)
$$

In combination with the renormalization condition, this subtraction can be implemented by a shift in the bare parameters of the theory

$$
\begin{array}{r}
m_{b}^{2}+\frac{\lambda_{b}}{16 \pi^{2}} \frac{\Lambda^{2}}{a^{2}(t)}=m_{r}^{2}\left[1+\frac{\lambda_{b}}{16 \pi^{2}} \ln (\Lambda / \kappa)\right] \\
\lambda_{b}=\frac{\lambda_{r}}{1-\frac{\lambda_{r}}{16 \pi^{2}} \ln (\Lambda / \kappa)} .
\end{array}
$$

The shift is time independent as long as the cutoff $\Lambda$ and the renormalization scale $\kappa$ are implemented in terms of the physical momentum [29]

$$
\begin{array}{r}
\Lambda=\Lambda_{0} a(t) \\
\kappa=\kappa_{0} a(t)
\end{array}
$$

where $\Lambda$ and $\kappa$ are comoving and $\Lambda_{0}$ and $\kappa_{0}$ are physical quantities. The renormalized mode function equation is

$$
\left(\frac{d^{2}}{d t^{2}}+3 \frac{\dot{a}(t)}{a(t)} \frac{d}{d t}+\frac{k^{2}}{a^{2}(t)}-m_{r}^{2}+\frac{\lambda_{r}}{2} G_{S}(t, t)\right) f_{k}(t)=0
$$

In following sections we will drop the renormalization subscripts for clarity and it is to be understood that we are working with renormalized quantities.

\section{Initial Conditions}

The quantum field is assumed to be initially in a state of thermal equilibrium. In this model with a tachyonic tree-level mass, symmetry is restored by finite temperature corrections. The initial effective mass $m_{e f f}^{2}$ is the solution of the (renormalized) equation

$$
m_{e f f}^{2}=-m^{2}+\frac{\lambda}{4 \pi^{2}} \int_{0}^{\Lambda} k^{2} d k\left(\frac{\operatorname{coth} \frac{\beta \sqrt{k^{2}+m_{e f f}^{2}}}{2}}{2 \sqrt{k^{2}+m_{e f f}^{2}}}-\frac{1}{2 k}+\theta(k-\kappa) \frac{m_{e f f}^{2}}{4 k^{3}}\right) .
$$

In the high temperature and small- $\lambda$ limit this yields

$$
m_{e f f}^{2}=-m^{2}+\frac{\lambda T^{2}}{24}
$$

which is a result familiar in finite-temperature field theory 13.

In an expanding FRW Universe, exact thermal equilibrium will only persist for conformally invariant fields. If the expansion rate is small relative to internal collisional processes of the field then there is an approximate notion of equilibrium [32. This is evidenced by transforming to conformal time, $\eta$, defined by 


$$
d t=a(\eta) d \eta
$$

and performing a mode redefinition

$$
\tilde{f}_{k}(\eta)=f_{k}(\eta) a(\eta)
$$

The conformal mode function equation is now

$$
\left(\frac{d^{2}}{d \eta^{2}}+k^{2}+a^{2}(\eta)\left(m^{2}+\frac{\lambda}{2} G(\eta, \eta)\right)\right) \tilde{f}_{k}(\eta)=0
$$

If the Universe is slowly expanding, a WKB-type solution is appropriate and a lowadiabaticity truncation of the instantaneous WKB frequency is sufficient. The zerothadiabatic order solution to equation (2.24) is given by

$$
\begin{gathered}
\tilde{f}(\eta)=\frac{1}{\sqrt{2 w_{k}}} e^{-i \int^{\eta} w_{k} d \eta^{\prime}}, \\
w_{k}^{2}=k^{2}+a(\eta)^{2} m_{e f f}^{2} .
\end{gathered}
$$

This leads to the following initial conditions for the mode functions in cosmic time $\mathrm{t}$

$$
\begin{array}{r}
f_{k}(0)=\frac{1}{a(0) \sqrt{2 w_{k}(0)}} \\
\dot{f}_{k}(0)=\left(-\frac{a(0)}{a(0)}-i w_{k}(0)\right) f_{k}(0) .
\end{array}
$$

Slow expansion assumes that the natural frequency of the kth mode is faster than the expansion rate of the Universe,

$$
w_{k}(0) \gg \frac{1}{2 \tau}
$$

The adiabatic equilibrium approximation would fail for the lowest $\mathrm{k}$ modes. However with high temperature initial conditions the low $\mathrm{k}$ modes are not a dominant part of the spectrum.

\section{Numerical Parameters}

The model described by Eq. 2.8) and (2.6) is characterized by 6 parameters,

$$
(m, \tau, \lambda, T, \Lambda, \kappa)
$$

We choose units of energy in which

$$
m^{2}=1.0
$$

The initial rate of expansion or the initial Hubble constant is controlled by $\tau$, 


$$
H(0)=\frac{\dot{a}(0)}{a(0)}=\frac{1}{2 \tau}
$$

In the simulations we conducted the range of the $\tau$ parameter was

$$
0.01 \leq \tau \leq 100
$$

and the self-coupling is strong,

$$
\lambda=0.1
$$

The initial temperature $T$ is chosen so that the value of the initial effective mass $m_{\text {eff }}^{2}(0)$ is of order unity. More specifically we choose

$$
\begin{aligned}
m_{\text {eff }}^{2}(0)=-1.0+\frac{\lambda}{24} T^{2} & =.607 \\
T & =20.0
\end{aligned}
$$

The values of the initial effective mass $m_{\text {eff }}^{2}(0)$, the coupling $\lambda$ and expansion rate $\tau$, were chosen so that the simulations of the phase transition completed on numerically accessible timescales. Due to the renormalization scheme, both the comoving cutoff $\Lambda$ and the comoving renormalization scale $\kappa$ increase with the scale factor. The initial value

$$
\Lambda_{0}=340
$$

was chosen as the lowest value that maintained cutoff independence of the initial effective mass. Insensitivity to the cut-off in the dynamical simulations was verified by doubling $\Lambda_{0}$ and observing no change in the output plots of the time-dependent effective mass. The initial value of the renormalization scale

$$
\kappa_{0}=1.0
$$

was chosen so that the renormalization scale was always above the maximum momentum of the unstable modes. The coupled, nonlinear system of mode function equations with the given initial conditions was solved numerically using an adaptive stepsize, fifth-order Runge-Kutta code. Mode integrals were performed using a simple simpson rule with a uniform momentum binning

$$
\begin{aligned}
& k=n k_{b i n}, \\
& k_{b i n}=\frac{2 \pi}{L_{0}},
\end{aligned}
$$

where $L_{0}=100.0$ is the effective size of the system and $\mathrm{n}$ is the total number of modes. Insensitivity to the momentum binning was verified by reducing $k_{b i n}$ and observing no change in output. The number of modes $n$ varied from $10^{4}$ to $10^{5}$. Run times varied from hours to days on a DEC $500 \mathrm{MHz}$ workstation which corresponds to dynamical time scales of $t=2$ to $t=100$. 


\section{Analysis}

The results of a typical simulation are shown in Figs. 1 and 2. In Fig. 1 the renormalized effective mass is shown as a function of cosmological time for quench parameter $\tau=1.0$. The phase transition begins when $M_{\text {eff }}^{2}$ first becomes negative. When $M_{\text {eff }}^{2}$ is negative, modes with physical momentum $\frac{k}{a} \leq M_{\text {eff }}$ have imaginary frequencies and begin to grow. This indicates the onset of the spinodal instability which is characteristic of a second-order phase transition. In the early stages of the phase transition, the evolution of the effective mass is dominated by the redshifting of stable modes and the effective mass decreases. As the phase transition proceeds, more modes redshift into the unstable momentum band and the amplitude of unstable modes continues to grow. Eventually, the redshift of stable modes balances the growth of unstable modes and the effective mass increases. As the effective mass passes through zero from below, the field reaches the spinodal point. Beyond the spinodal point all modes are stable. As the effective mass continues to grow, non-linear thermal and other collisional processes are expected to be important and the Hartree-Fock approximation of the dynamics of the two-point correlation function breaks down (see for example [19]).

In Fig. 2 we show the Fourier space structure factor

$$
S(k, t) \equiv k^{2} G_{k}(t, t)
$$

at various times during the phase transition. As argued in the next section, defects and domains can be identified in the low-k structure of $S(k, t)$. As the phase transition begins, $S(k, t)$ develops a peak at low $\mathrm{k}$. As the phase transition proceeds, this peak grows in amplitude and redshifts until at late times it completely dominates the infrared portion of the spectrum.

\section{DEFECT DENSITY}

We have argued that the dynamics of a phase transition of a quantum field may be approximated by the dynamics of the two-point function at least for times less than the spinodal time. Using the two-loop 2PI equations of motion we obtained a numerical solution for the evolution of the two-point function. To observe the formation of correlated domains and topological defects it is necessary to identify these structures from the form of the two-point function.

The identification of topological defects from the underlying quantum dynamics of the two-point function is a complicated problem. Intuitively, the existence of well-defined correlated domains of true vacuum at the completion of the phase transition is similar to the domains that form in a condensed matter system like a ferromagnet. However, our system is described by a quantum field theory and the existence of a classical configuration of domains requires a quantum-to-classical transition, of which decoherence is an essential condition. The extraction of a positive-definite probability distribution, $P[\Phi]$, from the density matrix requires a quantitative treatment of the quantum-to-classical transition. Classical defect solutions would then appear in field configurations drawn from $P[\Phi]$ [12]. 
In this research our focus is not on the extraction of classical defects from the quantum system but instead on the long wavelength modes that determine the size of correlated domains and, therefore, the average defect separation. The long wavelength modes interact with an environment of short wavelength and thermal fluctuations that destroys quantum coherence among the long wavelength modes and results in a finite correlation length for the system [33]. While quantum fluctuations are indeed important to the description of phenomena within the scale of one domain, beyond this scale we may hope to treat the modes as classical. With this abbreviated estimate of decoherence we show that the existence and size of correlated domains is indicated by an infrared peak in the power spectrum of the equal-time momentum-space two-point function.

For a free field theory quenched into the unstable region by an instantaneous change in the sign of the square of the mass at $t=t_{0}$ it is possible to obtain analytic expressions for the equal-time momentum space two-point function [20]. After the quench, the momentum space structure function $k^{2} G(k, t)$ has a strong maximum at the value

$$
k_{\max } \sim \sqrt{\frac{m_{f}}{2 t}}
$$

The real space Fourier transform of this function (normalized to unity at $\left|\vec{r}-\overrightarrow{r^{\prime}}\right|=0$ ) is

$$
G\left(\left|\vec{r}-\overrightarrow{r^{\prime}}\right|, t\right) \sim e^{-\frac{m_{f}\left(\left|\vec{r}-\vec{r}^{\prime}\right|\right)^{2}}{8 t}}
$$

This form of the equal-time correlation function is common in condensed matter systems [34]. From the equal-time real space correlation function we can identify the correlation length

$$
\xi(t) \sim \sqrt{\frac{8 t}{m_{f}}} \sim \frac{1}{k_{\max }}
$$

In analogy with the free field theory, we assume the domain size is proportional to the size of the maximum of the momentum space structure function.

Even when the density matrix of the quantum field has decohered into a positive-definite probability distribution for an ensemble of classical field configurations, the identification of topological defect structures is a complicated task. In a classical field configuration and when defects are well-formed (so that the width of the defect is much smaller than the typical defect spacing), it is possible to identify zeros of the classical field configurations with topological defects. In a model with a global $O(n)$ symmetry in $n$ spatial dimensions, and when the field probability distribution is Gaussian, a formula for the ensemble average density of field zeros was given by Halperin [35] and derived explicitly by Liu and Mazenko [36]:

$$
\begin{gathered}
\rho(t)=C_{n}\left(\frac{G^{\prime \prime}(0, t)}{G(0, t)}\right)^{n / 2} \\
C_{1}=\frac{1}{\pi}, C_{2}=\frac{1}{2 \pi}, C_{3}=\frac{1}{\pi^{2}}
\end{gathered}
$$


For a single scalar field in 3 spatial dimensions Eq. (3.4) with $n=1$ is valid as the ensembleaveraged density of zeros along a one-dimensional section of the field. The validity of the Gaussian approximation is further discussed in [37.

A zero of the classical field configuration does not uniquely identify a topological defect. Thermal fluctuations give a large number of zeros of the field configuration on small scales [38]. The zeros of the field configuration which are not associated with different defects lead to an ultraviolet momentum divergence in the expression for the zero density, Eq. (3.4). To make physical sense of the zero formula, a coarse graining of the field configuration is needed. The spacing of defects and the size of correlated domains is determined by long wavelength excitations and if zeros of the field configuration are to accurately count defects, it is necessary to apply a coarse-graining that removes the short-wavelength excitations caused by quantum and thermal fluctuations. To effect this coarse-graining in Eq.(3.4) we impose a spatial momentum cut-off at the upper edge of the unstable momentum band. Only the unstable modes will contribute to the domain size.

In principle it is possible that the length scales set by $k_{\max }(t)$ and by $\rho(t)$ are different. When the structure factor $k^{2} G(k, t)$ is very strongly peaked about $k_{\text {max }}$ we can approximate the unstable portion of the spectrum as

$$
k^{2} G(k, t) \sim \delta\left(k-k_{\max }\right)
$$

the zero density for a single scalar field in 3 dimensions is then

$$
\rho(t)=\frac{1}{\pi}\left(\frac{\int k^{4} G(k, t)}{\int k^{2} G(k, t)}\right)^{3 / 2} \sim k_{\max }(t)^{3} .
$$

For the parameters used in the simulation and at late times, the scales set by the zero density and by the domain size differ only by a numerical factor of order unity. Using the linear model introduced in Sec. $\mathrm{V}$, evidence that the growth of domains is characterized by one scale is shown in Fig. 5. Additional evidence for a "one-scale" model was given in [39] in the context of the dynamics of a classical field phase transition in $1+1$ dimensions.

For very weak coupling not considered here and when a peak is no longer evident in $S(k, t)$, the one-scale approximation will fail and it is possible in principle for the defect density to depart significantly from one per correlation volume [22].

\section{CRITICAL SCALING}

The evolution of the two-point function and the extraction of correlated domains and topological defects allows a first-principles analysis of the mechanisms important for topological defect formation. In light of the "freeze-out" scenario it is interesting to compare the initial size of correlated domains for scale factors $a_{\tau}(t)$ with different expansion rates $\tau$. To quantify the dependence of the initial size of correlated domains on the quench rate $\tau$ of the phase transition, we compare the size of domains for different values of the parameter $\tau$. As discussed above, the average size of a domain is proportional to the maximum in the infrared portion of $S(k, t)$. We compare the maximum $k_{\max }$ for different values of $\tau$ and at two distinct sets of times during the phase transition. The first set of domains is measured 
when the square of the effective mass reaches a minimum value. This provides an early-time measure of the size of domains. The results are shown in Fig. 3. The second set of domains is measured when the square of the effective mass reaches a local maximum value after the phase transition. This provides a late-time measure of the size of domains. The results are shown in Fig. 1 and Fig 5 . For slow quench rates $(\tau \geq 1.0)$, at both early and late times, the dependence of $k_{\max }$ on the quench rate $\tau$ is well approximated by a power law,

$$
k_{\max }(\tau) \sim \tau^{-0.35}
$$

For fast quench rates $(\tau<1.0)$, the dependence of $k_{\max }$ on the quench rate $\tau$ is well approximated by a power law,

$$
k_{\max }(\tau) \sim \tau^{-0.28}
$$

The origin of these exponents and the difference between slow and fast quenches is discussed in the following section.

\section{A. The Freeze-Out Scenario}

In Zurek's scenario the "frozen" correlation length and therefore the initial size of correlated domains scale as a power law of the quench time $\tau$ as

$$
\xi_{\text {freeze }} \sim \tau^{\frac{\nu}{1+\mu}}
$$

where $\mu$ and $\nu$ are the equilibrium critical exponents for the correlation length and relaxation time respectively. The correlation length and relaxation time are identified as, respectively, the length and time scales that characterize the equilibrium behavior of the propagator near the critical temperature [40]. Under the scaling hypothesis, the equal-time propagator is written

$$
G_{k}=k^{-(2-\eta)} F[h(\epsilon) k] .
$$

Here $\epsilon$ is the reduced temperature, Eq. (1.4), $\eta$ is a critical exponent (not to be confused with conformal time) and $F$ is a dimensionless function. The finite-temperature equilibrium correlation length is

$$
\xi(\epsilon)=h(\epsilon) \sim \epsilon^{-\nu}
$$

Similarly the two-time propagator is

$$
G_{\omega, k}=\omega^{\alpha} F\left[h^{\prime}(\epsilon) \omega, h(\epsilon) k\right]
$$

so that the relaxation time

$$
\tau \sim h^{\prime} \sim \epsilon^{-\mu}
$$

In the Hartree-Fock approximation the equilibrium propagator is 


$$
G_{\omega, k} \sim \frac{1}{\omega^{2}-k^{2}-m_{e f f}^{2}} \operatorname{coth}\left(\frac{\sqrt{k^{2}+m_{e f f}^{2}}}{2} \beta\right)
$$

where the effective mass is given by the equilibrium value Eq. (2.21). Near the critical point

$$
m_{e f f}^{2}(\epsilon)=A \epsilon
$$

where $\mathrm{A}$ is a constant independent of temperature. The scaling behavior of the propagator is as $\frac{\omega}{\sqrt{\epsilon}}$ and $\frac{k}{\sqrt{\epsilon}}$ and therefore the critical exponents for the theory are

$$
\mu=\nu=\frac{1}{2}
$$

The critical exponents derived for this quantum field theory in 4 spacetime dimensions are the same as the critical exponents of those of a classical Ising model in 4 space dimensions since, after the Euclidean continuation $t \rightarrow i \beta$, they are in the same universality class. The finite size $\beta$ of the Euclideanized time dimension can alter the critical behavior of the quantum field [41]. At high temperature or near a second-order phase transition equilibrium quantum systems in $d$ spacetime dimensions undergo dimensional reduction and behave with the critical exponents of classical systems in $d-1$ space dimensions [42]. The dimensional crossover can be parameterized by

$$
z=\xi_{T} T
$$

where $\xi_{T}$ is the equilibrium correlation length and $\mathrm{T}$ is the temperature. The effective critical exponents are functions of $z$ so that $z=0$ corresponds to the quantum case and $z=\infty$ corresponds to the classical case. Intuitively, dimensional crossover occurs in a secondorder phase transition when the correlation length $\xi_{T}$ is much larger than the Euclidean compactified dimension $\beta=1 / T$. The quantitative effect of dimensional reduction on the critical exponents has been calculated in detail in 42 where plots of $\nu_{\text {eff }}(z)$ and $\mu_{\text {eff }}(z)$ may be found. In the extreme dimensionally-reduced case, when $z \rightarrow \infty$, the critical exponents change to

$$
\mu=\nu \approx 0.64
$$

However, in our model, the dynamics of the system and in particular the "freeze-out" of the correlation length prevent $z$ from becoming too large and the actual critical exponents lie somewhere between the four dimensional case Eq. (4.10) and the three dimensional case Eq. (4.12). The "freeze-out" prediction for the initial scale of the density of topological defects, Eq. (4.3), is relatively insensitive to this change in critical exponents and varies from

$$
\xi_{\text {freeze }} \sim \tau^{0.33}
$$

in the four dimensional case to

$$
\xi_{\text {freeze }} \sim \tau^{0.38}
$$


in the three dimensional case. Our observation of the power law exponent of 0.35 in the numerical simulations for slow quenches, $\tau \geq 1.0$, (Fig. 3 and Fig. 4) is consistent with these cases.

The calculation of the equilibrium critical exponent for the relaxation time depends on the dynamics of the mode functions for the the low $\mathrm{k}$ modes near the critical point and in particular whether the dynamics is overdamped or underdamped. Near the critical point, $m_{\text {eff }}^{2} \approx 0$, and the mode function equation is approximately

$$
\left(\frac{d^{2}}{d t^{2}}+3 \frac{\dot{a}\left(t_{c}\right)}{a\left(t_{c}\right)} \frac{d}{d t}+\frac{k^{2}}{a^{2}\left(t_{c}\right)}\right) f_{k}(t)=0 .
$$

The critical time $t_{c}$ can be estimated as the time when the equilibrium effective mass, Eq. (5.6), goes to zero,

$$
t_{c}=\frac{2}{3} \tau
$$

and the value of the scale factor at $t_{c}$ is

$$
a\left(t_{c}\right)=\left[\frac{t_{c}+\tau}{\tau}\right]^{\frac{1}{2}} \approx 1.3
$$

The mode function equation near the critical point Eq. (4.15) is that of a damped harmonic oscillator with natural frequency $\omega_{0}=\frac{k}{a\left(t_{c}\right)}$ and damping constant $\Gamma$ where

$$
\Gamma=3 H\left(t_{c}\right) \approx \frac{1}{\tau}
$$

The modes for which $\omega_{0}>\Gamma$ or

$$
k>\frac{1.3}{\tau}
$$

are underdamped. The range of wavenumbers $k$ for the modes that determinex the size of correlated domains also depends on $\tau$. Eq. (4.19) is a condition on $\tau$ such that when

$$
\tau>\tau_{*}
$$

the modes responsible for domain formation are underdamped and $\tau_{*}$ is to be determined. For the underdamped case we expect $k_{\max } \sim \tau^{-\frac{1}{3}}$. Using $k_{\max }$ as a representative wavevector of the modes responsible for domains, Eq. (4.19) implies the underdamped condition

$$
\tau_{*} \sim 1
$$

Slow quenches with $\tau \geq 1.0$ are therefore underdamped. Fast quenches are overdamped and the dynamics of the mode functions will be dominated by the first time derivative. In the overdamped case, the critical exponent, $\mu$, now assumes the non-relativistic value

$$
\mu_{\text {overdamped }}=2 \mu_{\text {underdamped }}
$$


Due to the uncertainties of dimensional reduction, in the overdamped case, the initial scale of the density of topological defects ranges from

$$
\xi_{\text {freeze }} \sim \tau^{0.25}
$$

for the four dimensional critical exponents to

$$
\xi_{\text {freeze }} \sim \tau^{0.28}
$$

in the three dimensional case. This is in good agreement with the power law exponent of 0.28 measured for overdamped, fast quenches $(\tau<1.0)$ shown in Fig. 5 .

In both the overdamped and underdamped cases, the prediction of the "freeze-out" proposal for the power law exponent of the scaling of the initial defect density with the quench rate appears to be in excellent agreement with the numerical simulations.

\section{B. Early-Time Linear Approximation}

If the initial size of correlated domains and the initial density of topological defects are determined by processes occurring very near the critical point, as claimed in the "freeze-out" proposal, then the power law scaling of domains with the quench parameter $\tau$ observed in the simulation of the Hartree-Fock mode function Eq. (2.19) will also appear in a linear approximation. A linear amplitude approximation to the full mode function equation is valid for slow quenches and only near the onset of the spinodal instability, before the unstable modes have grown appreciably and back reaction is important. Consider the linear equation

$$
\left[\frac{d^{2}}{d t^{2}}+3 \frac{\dot{a}}{a} \frac{d}{d t}+\frac{K^{2}}{a^{2}}-m^{2}\right] F_{k}=0
$$

where

$$
K^{2}=k^{2}+k_{0}^{2}
$$

and $k_{0}$ is related to the initial temperature

$$
k_{0}^{2}=\frac{\lambda}{24} T_{0}^{2}
$$

In conformal time, we have

$$
\eta^{2}=4 \tau(t+\tau)
$$

with conformal modes, $f_{k}$, defined by

$$
f_{k} \equiv F_{k} a .
$$

Eq.(4.25) may be rewritten in conformal time as

$$
\left[\frac{d^{2}}{d \eta^{2}}+K^{2}-\frac{m^{2} \eta^{2}}{4 \tau^{2}}\right] f_{k}=0 .
$$


This equation can be solved exactly in terms of parabolic cylinder functions. However, a simpler solution is obtained for times near the critical point. We further approximate it by

$$
\begin{array}{r}
{\left[\frac{d^{2}}{d \eta^{2}}-\frac{m K}{\tau}\left(\eta-\eta_{k}\right)\right] f_{k}=0} \\
\eta_{k} \equiv \frac{2 K \tau}{m}
\end{array}
$$

The properly normalized solution with vacuum boundary conditions before the instability is

$$
f_{k}=i \sqrt{\frac{2 x}{3 \pi}} K_{1 / 3}\left[-\frac{2}{3} \sqrt{\frac{m K x^{3}}{\tau}}\right]
$$

where

$$
x=\eta-\eta_{k}
$$

and $K_{1 / 3}$ is a modified Bessel function.

We now use the analytic solution Eq. (4.33) to examine the dependence of the position of the peak in the Fourier space structure factor $k^{2} G(k, t)$ on the quench parameter $\tau$. The location of this peak $k_{\max }(t)$ redshifts throughout the phase transition, moving towards lower momentum as the domains coarsen. In order to compare domains formed in the linear model with those of the Hartree evolution we compare the position of the peaks at very early times when a peak is first identifiable in $S(k, t)$. Plots of $S(k, t)$ with the analytic modes were made with Mathematica. The results are shown in Fig. 7. The scaling of domains with the quench parameter $\tau$ with the same power law exponent as seen in the full numerical simulation for slow quenches is evident.

\section{DISCUSSION AND CONCLUSION}

Using the Hartree-Fock approximation to the equations of motion for the two-point function of a quantum scalar field undergoing a phase transition in a $3+1$ dimensional spatially flat, radiation-dominated FRW Universe we have shown that the size of correlated domains, measured as the maximum of the peak of the infrared part of the spectrum of $k^{2} G(k, t)$, scales as a power of the quench rate $\tau$ as

$$
\xi_{\text {domains }} \sim \tau^{0.35}
$$

for slow, underdamped quenches $(\tau \geq 1.0)$ and

$$
\xi_{\text {domains }} \sim \tau^{0.28}
$$

for fast, overdamped quenches $(\tau<1.0)$. The observed power law scaling of correlated domains is quantitatively consistent with the "freeze-out" hypothesis. In both overdamped and underdamped cases, the value of the power law exponent extracted from the Hartree-

Fock evolution of the two-point function is in good agreement with the value calculated in the 
"freeze-out" scenario using the critical scaling exponents for a $\Phi^{4}$ theory in the Hartree-Fock approximation.

To further explore the behavior of the quantum system near the critical point we introduced an approximate linear model valid for slow quenches and short times after the onset of spinodal instability. In this linear model, the size of correlated domains scales with the quench rate $\tau$ to the same power as observed in the underdamped numerical simulations and predicted by the "freeze-out" proposal. This provides analytical evidence for the "freeze-out" hypothesis. A similar analytic model was introduced in the context of a $1+1$ dimensional classical condensed matter system in [43]

In contrast to previous approaches to the problem of defect formation in quantum field theory, we have allowed for the process of back reaction, which permits the quantum field to exit the region of spinodal instability. The power law exponent is the same whether it is measured at very early times with the analytic linear model (Fig. 7) or, in the numerical simulations, at early times in the middle of the spinodal region at the minimum value of $M_{\text {eff }}^{2}$ (Fig. 3) or, at late times in the stable region at the maximum value of $M_{\text {eff }}^{2}$ (Fig. (4). This is consistent with the idea that the relevant processes for the growth of domains occur near the critical point. However, back reaction is essential to the freezing in of the value of the power law exponent and to the "freeze-out" hypothesis. Although the power law is accurately recorded in the early time analytical model, if back reaction is ignored and the linear model is (incorrectly) extrapolated to late times, the predicted scaling exponent is different

$$
k_{\max } \sim \tau^{-\frac{1}{2}}
$$

The results derived from the numerical simulation of the mode function equation with back reaction and the analytical solution around the critical point are evidence supporting the first verification of the "freeze-out" scenario in 3 spatial dimensions and in a realistic system relevant to the early Universe. It is at first surprising that arguments based on classical equilibrium critical scaling apply in the context of dynamical quantum field theory. A possible explanation rests with the high temperature initial conditions and the properties of classical $\lambda \Phi^{4}$ theory in FRW spacetime. Since the initial temperature is much higher than the initial effective mass

$$
T_{0}>>m_{e f f}(0)
$$

the field is approximately conformally invariant. In an FRW Universe, a conformally invariant field initially in thermal equilibrium will remain in equilibrium at a redshifted temperature

$$
T(t)=\frac{T_{0}}{a(t)} .
$$

A well-known example is the redshifting of the blackbody spectrum of the cosmic microwave background radiation as the Universe expands. To illustrate the approximate conformal invariance in our model we compare the dynamical effective mass from the simulations with the effective mass of a theory with the equilibrium redshifted temperature of Eq. (5.5) 


$$
M_{e q}^{2}=-m^{2}+\frac{\lambda}{24} \frac{T_{0}^{2}}{a^{2}(t)} .
$$

The results are shown in Fig. 8. Only after the onset of the spinodal instability do the equilibrium and dynamical effective mass differ significantly. The approximate conformal invariance of the theory means that the finite-temperature effective potential and the critical behavior derived from it are approximately valid until times near the onset of the spinodal instability. The high temperature initial conditions also offer a possible explanation for the observed classical behavior of the system. At such high temperature, thermal fluctuations dominate over quantum vacuum fluctuations.

The agreement in the value of the power law exponent between the microscopic evolution equations of the quantum field theory and phenomenological critical scaling supports the contention that quantum critical systems in the early Universe share, in certain circumstances, many of the properties of their classical counterparts. It would be very interesting to further explore these connections. To do so, however, it is necessary to go beyond the strong coupling and high temperature conditions used in this research.

The results derived in this paper represent a preliminary step in a first-principles approach to the calculation of the topological defect density immediately following the completion of a second-order phase transition in the early Universe. Our work uses a microscopic quantum field theory and incorporates realistic initial conditions and a physical quench mechanism. However, the domain wall defects formed in this model are inconsistent with cosmological observations. It is not difficult in principle to apply the methods used in this paper to more cosmologically realistic theories such as cosmic string models. The analytical and numerical evidence for the power law scaling of the domains is expected to hold in a more realistic model.

The formalism of the 2PI-CTP effective action and related techniques can be used to probe more general questions related to the physical aspects of quantum critical dynamics [28]. To observe and isolate quantum processes it is necessary to relax the assumption of high temperature initial conditions, allowing quantum vacuum fluctuations to dominate over thermal fluctuations. Also important are the detailed properties of the system-bath interaction. A system-bath interaction such as proposed in 19 could address such issues as critical slowing down and the role of dissipation and noise. Such work is in progress.

\section{ACKNOWLEDGMENTS}

This work is supported in part by the National Science Foundation under grant PHYS9800967. GJS would like to thank the National Scalable Computer Project at the University of Maryland for access to computing resources. 


\section{REFERENCES}

[1] A. Vilenkin and E. P. S. Shellard, Strings and Other Topological Defects, (Cambridge Univ. Press, Cambridge, UK 1994).

[2] T. W. B. Kibble, J. Phys. A9, 1387 (1976).

[3] A. H. Guth, Phys. Rev. D23, 347 (1981).

[4] W. H. Zurek, Nature 317, 505 (1985): W. H. Zurek Phys. Rep. 276, 178 (1996).

[5] G. E. Volovik "Superfluid ${ }^{3} \mathrm{He}$, Particle Physics and Cosmology", [Report No. condmat/9711031 , (1997).

[6] I. Chuang, R. Durrer, N. Turok, and B. Yurkee, Science 251, 1336 (1991); M. J. Bowick, L. Chandar, E. A. Schiff, and A. M. Srivastava, Science 263, 943 (1994).

[7] P. C. Hendry et al Nature 368, 315 (1994); C. Bauerle et al Nature 382, 332 (1995); V. M. Ruutu et al Nature 382, 334 (1996).

[8] P. Laguna and W. H. Zurek, Phys. Rev. Lett 78, 2519 (1997).

[9] A. Yates and W. H. Zurek, Phys. Rev. Lett 80, 5477 (1998).

[10] P. C. Hohenberg and B. I. Halperin, Rev. Mod. Phys. 49, 435 (1977).

[11] B. L. Hu, in Proceedings of the Third International Workshop on Thermal Fields and its Applications, CNRS Summer Institute, Banff, August 1993, edited by R. Kobes and G. Kunstatter (World Scientific, Singapore, 1994).

[12] F. Cooper, S. Habib, Y. Kluger and E. Mottola, Phys. Rev. D55, 6471 (1997).

[13] D. A. Kirzhnitz and A. D. Linde, Phys. Lett. 42B, 471 (1972).

[14] L. Dolan and R. Jakiw, Phys. Rev. D9, 639 (1974).

[15] S. Weinberg, Phys. Rev. Lett. 9 3357, (1974).

[16] S. K. Ma, "Modern Theory of Critical Phenomena", (Benjamin/Cummings, Reading, MA, 1976); J. Zinn-Justin, "Quantum Field Theory and Critical Phenomena", (Oxford Univ. Press, Oxford, UK 1996).

[17] G. F. Mazenko, W. G. Unruh and R. M. Wald, Phys. Rev. D31, 273 (1985).

[18] S. A. Ramsey and B. L. Hu, Phys. Rev. D56, 678 (1997); S. A. Ramsey, B. L Hu and A. M. Stylianopoulos, Phys. Rev. D57, 6003 (1998)

[19] E. Calzetta, Ann. Phys. 190, 32 (1989).

[20] D. Boyanovsky, D. Lee and A. Singh, Phys. Rev. D48, 800 (1993).

[21] A.J. Gill and R. J. Rivers, Phys. Rev D51, 6949 (1995).

[22] G. Karra and R. J. Rivers, Phys. Lett. B414, 28 (1997).

[23] M. Bowick and A. Momen, "Domain Formation in Finite-Time Quenches", [Report No. hep-ph/9803284.

[24] N. Goldenfeld in Formation and Interactions of Topological Defects edited by A. C. Davis and R. Brandenberger, (Penum Press, New York, 1995), [Report No. hepph/9411380].

[25] E. Calzetta and B. L. Hu, Phys. Rev. D35, 495 (1987).

[26] E. Calzetta and B. L. Hu, Phys. Rev. D37, 2878 (1988).

[27] J. M. Cornwall, R. Jackiw and E. Tomboulis, Phys. Rev. D10, 2428 (1974).

[28] E. Calzetta and B. L. Hu, in Heat Kernel Techniques and Quantum Gravity, Vol. 4 of Discourses in Mathematics and Its Applications, Winnipeg, 1994, edited by S. A. Fulling (Texas A\&M University Press, College Station, TX, 1995), [Report No. hepth/9501040. 
[29] D. Boyanovsky, D. Cormier, H.J. de Vega, R. Holman, A. Singh and M. Srednicki, Phys. Rev. D56, 1939 (1997).

[30] D. Boyanovsky, H. J. de Vega and R. Holman, Phys. Rev. D49, 2769 (1994).

[31] R. G. Root, Phys. Rev. D10, 3322 (1974).

[32] B. L. Hu, Phys. Lett. 108B, 19 (1982);123B, 189 (1983).

[33] B. L. Hu, J. P. Paz and Y. Zhang in The Origin of Structure in the Universe edited by E. Gunzig and P. Nardone (Kluwer Academic Publishers, Dordrecht, 1993).

[34] A. J. Bray, Adv. phys. 43, 357 (1994).

[35] B. Halperin in Physics of Defects edited by R. Balian, M. Kleman and J. P. Poirier (North-Holland, New York, 1981).

[36] F. Liu and G. F. Mazenko, Phys. Rev, B46, 5963 (1992).

[37] E. Calzetta and D. Ibaceta, in preparation.

[38] F. J. Alexander, S. Habib, and A. Kovner, Phys. Rev.E48, 4284 (1993).

[39] N. D. Antunes and L. M. A. Bettencourt, Phys. Rev. D55, 225 (1997).

[40] P. M. Chaikin and T. C. Lubensky, Principles of Condensed Matter Physics, (Camb. Univ. Press. New York, USA 1995).

[41] B. L. Hu and D. J. O'Connor, Phys. Rev. D36, 1701 (1987).

[42] D. O'Connor and C. R. Stephens, Int. J. Mod. Phys. A9, 2805 (1994); D. O'Connor and C. R. Stephens, Int. J. Mod. Phys. B12, 1379 (1998).

[43] J. Dziarmaga, "Density of kinks just after a quench in an underdamped system", [Report No. cond-mat/9803185. 


\section{FIGURES}

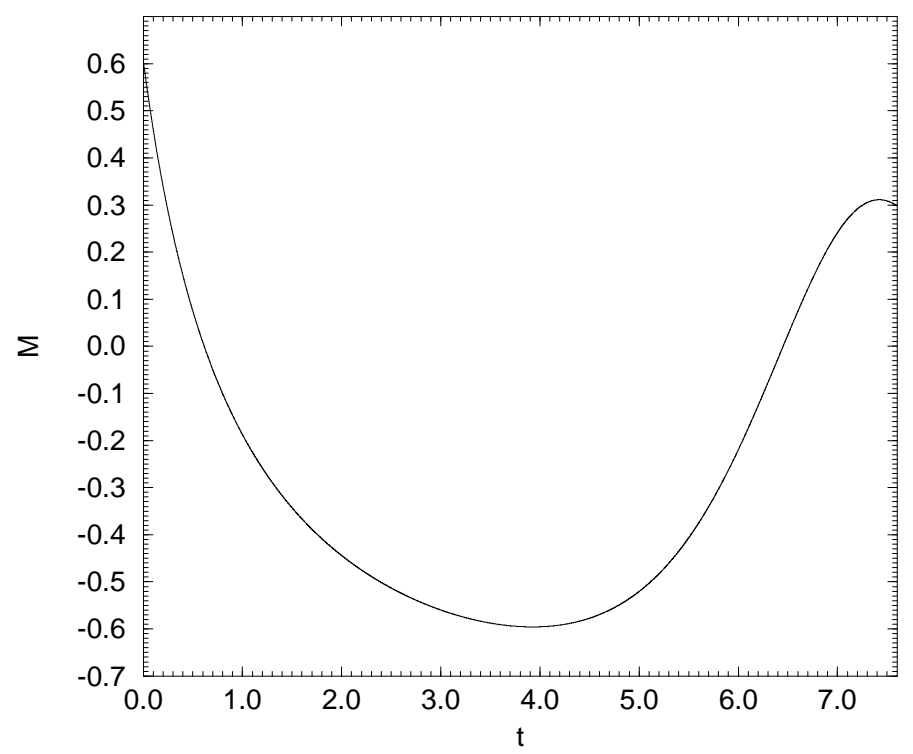

FIG. 1. Plot of the square of the (renormalized) effective mass $M=m_{\text {eff }}^{2}$ vs. cosmological time $t$ for quench parameter $\tau=1.0$.

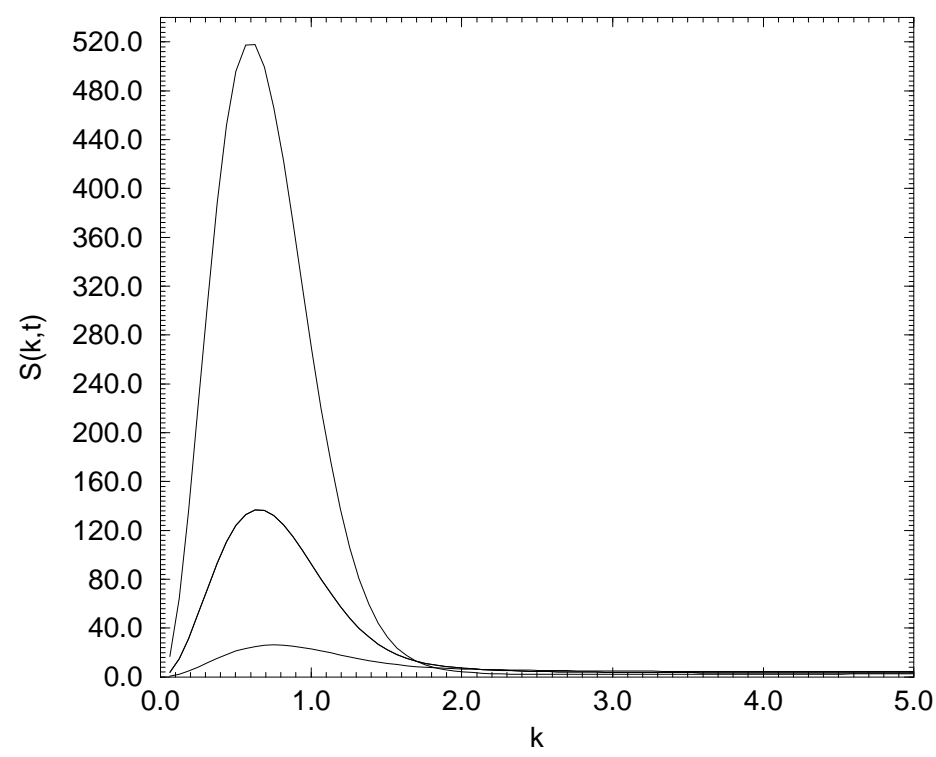

FIG. 2. Plot of the Fourier-space structure factor, $S(k, t)=k^{2} G(k, t)$, vs. comoving momentum $k$ at various times during the evolution for quench parameter $\tau=1.0$. The bottom curve is a snapshot of $S(k, t)$ at time $t=3.9$. The middle curve is a snapshot at $t=5.4$. The top curve is a snapshot at $t=6.9$. 


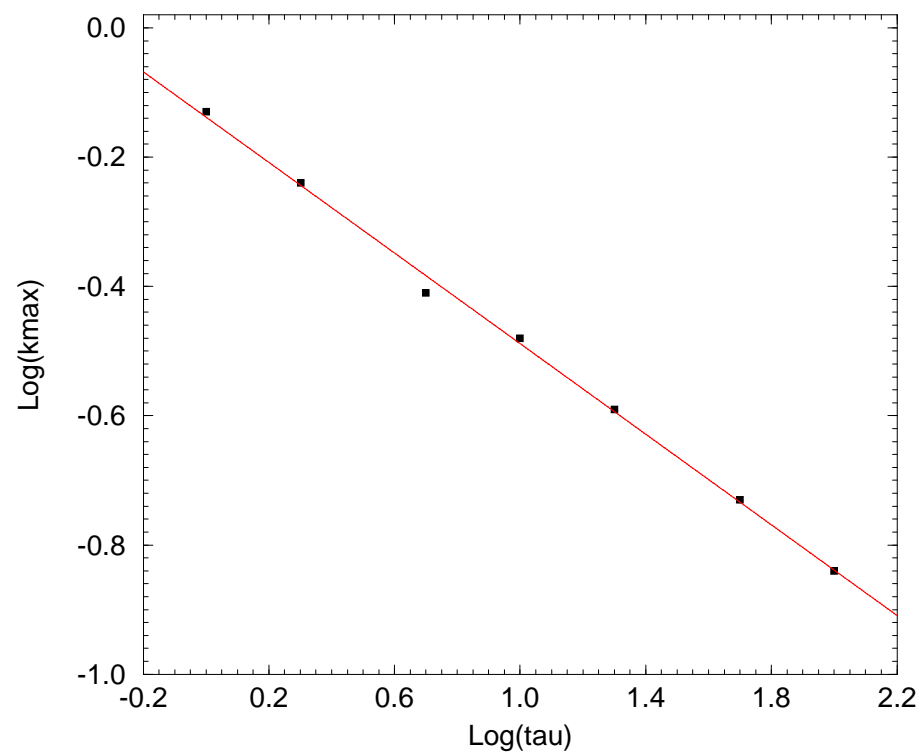

FIG. 3. Plot of $\log _{10}\left(k_{\max }\right)$ vs. $\log _{10}(\tau)$ in the case of slow, underdamped quenches $(\tau \geq 1.0)$ for domains formed early in the phase transition, at the time when the square of the effective mass reaches a minimum value. Filled squares are measurements from the numerical simulations. The solid line is a plot of the best-fit linear function to the data, $\log _{10}\left(k_{\max }\right)=-0.35 \log _{10}(\tau)-0.14$.

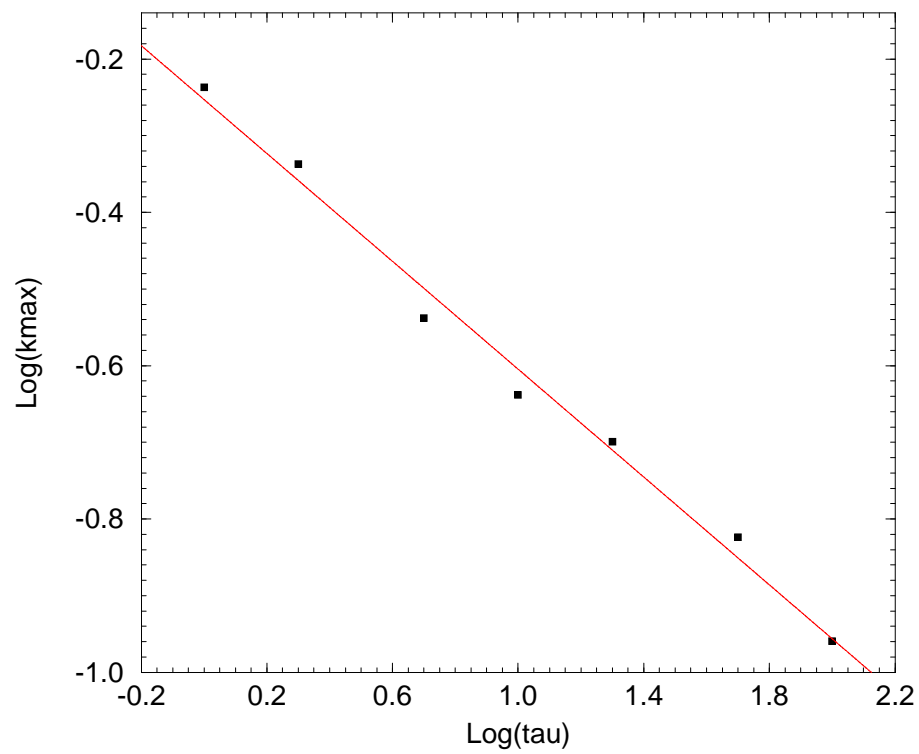

FIG. 4. Plot of $\log _{10}\left(k_{\max }\right)$ vs. $\log _{10}(\tau)$ in the case of slow, underdamped quenches $(\tau \geq 1.0)$ for domains formed late in the phase transition, at the time when the square of the effective mass reaches a local maximum value. Filled squares are measurements from the numerical simulations. The solid line is a plot of the best-fit linear function to the data, $\log _{10}\left(k_{\max }\right)=-0.35 \log _{10}(\tau)-0.26$. 


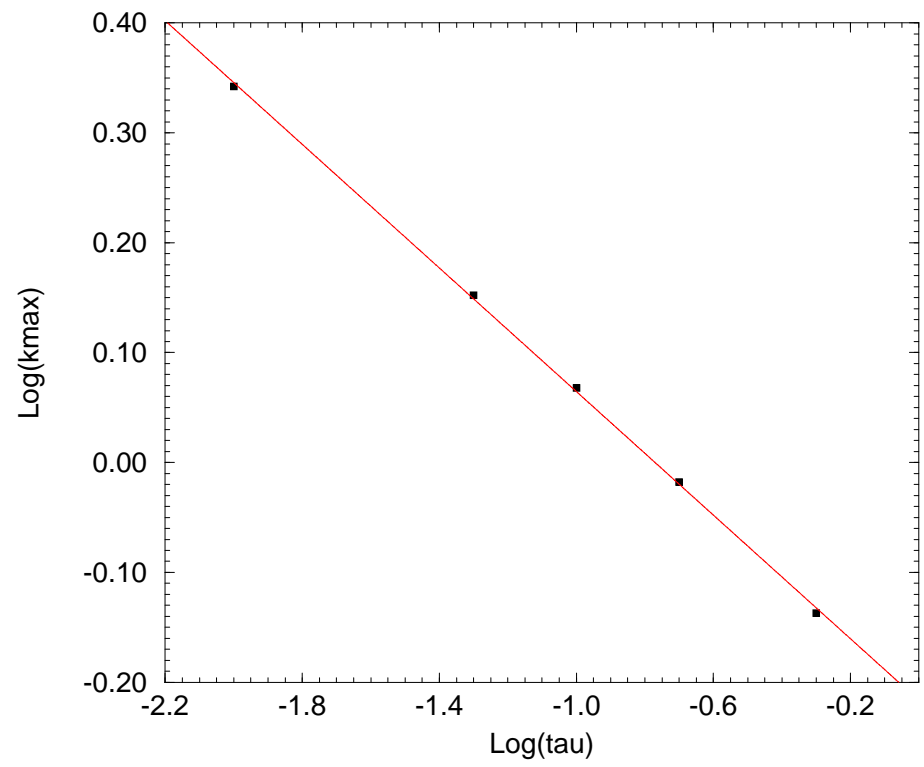

FIG. 5. Plot of $\log _{10}\left(k_{\max }\right)$ vs. $\log _{10}(\tau)$ in the case of fast, overdamped quenches $(\tau<1.0)$ for domains formed late in the phase transition, at the time when the square of the effective mass reaches a local maximum value. Filled squares are measurements from the numerical simulations. The solid line is a plot of the best-fit linear function to the data, $\log _{10}\left(k_{\max }\right)=-0.28 \log _{10}(\tau)-0.22$.

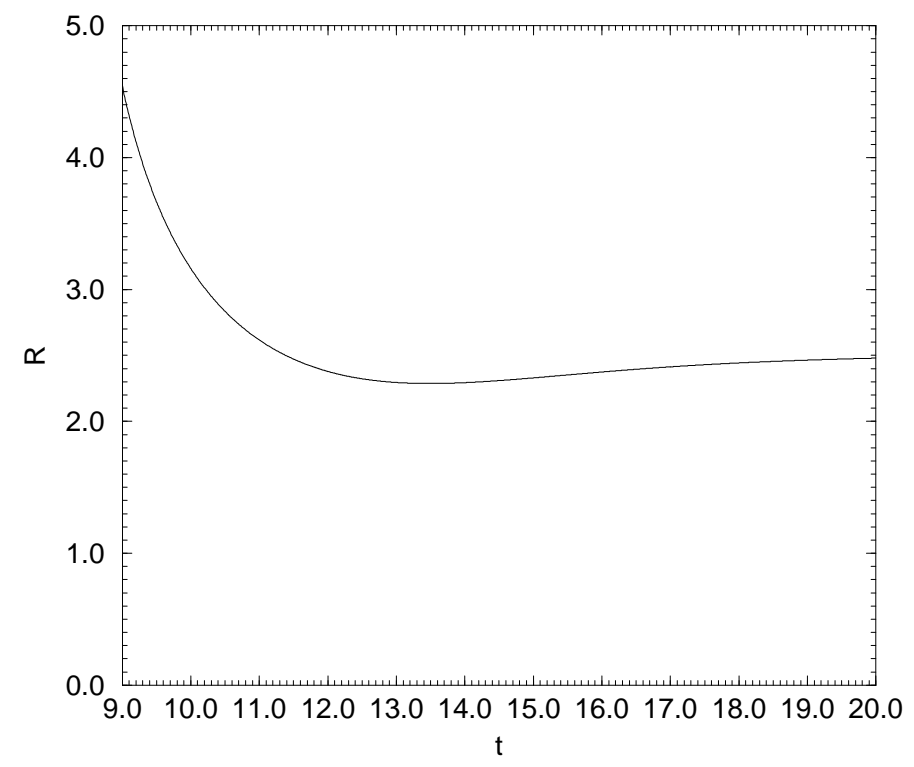


FIG. 6. Plot of the ratio, $R(t)=\frac{k_{\max }(t)}{\rho(t)}$, vs. cosmological time $t$ for quench parameter $\tau=10.0$. The defect density $\rho(t)$ was calculated using the analytic modes, Eq. (4.33), and the Halperin-Mazenko-Liu formula, Eq. (3.4), with a cutoff at the maximum momentum of the unstable band. The maximum $k_{\max }(t)$ in the structure function, $S(k, t)=k^{2} G(k, t)$, was also determined using the analytic modes, Eq.(4.33). At late times this ratio approaches a constant that is independent of the quench parameter.

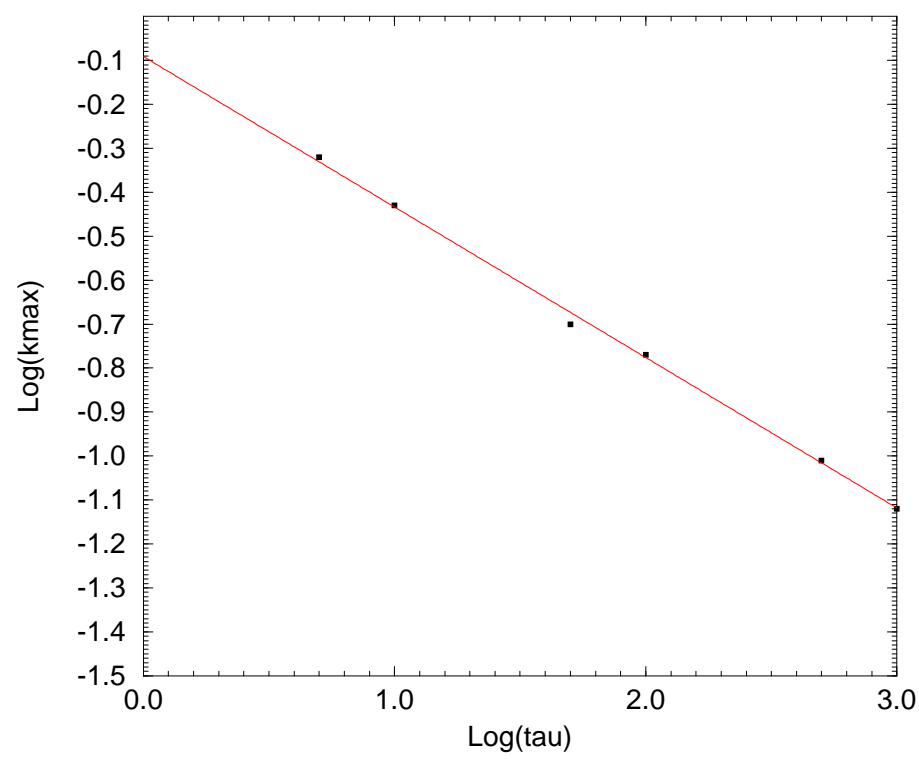

FIG. 7. Plot of $\log _{10}\left(k_{\max }\right)$ vs. $\log _{10}(\tau)$ in the analytic model, Eq. 4.33). The maximum, $k_{\max }$, was determined as soon as a peak was evident in the structure function, $S(k, t)$. Filled squares are measurements from plots of the analytic modes. The solid line is a plot of the best fit linear function to the data, $\log _{10}\left(k_{\max }\right)=-0.34 \log _{10}(\tau)-0.09$. 


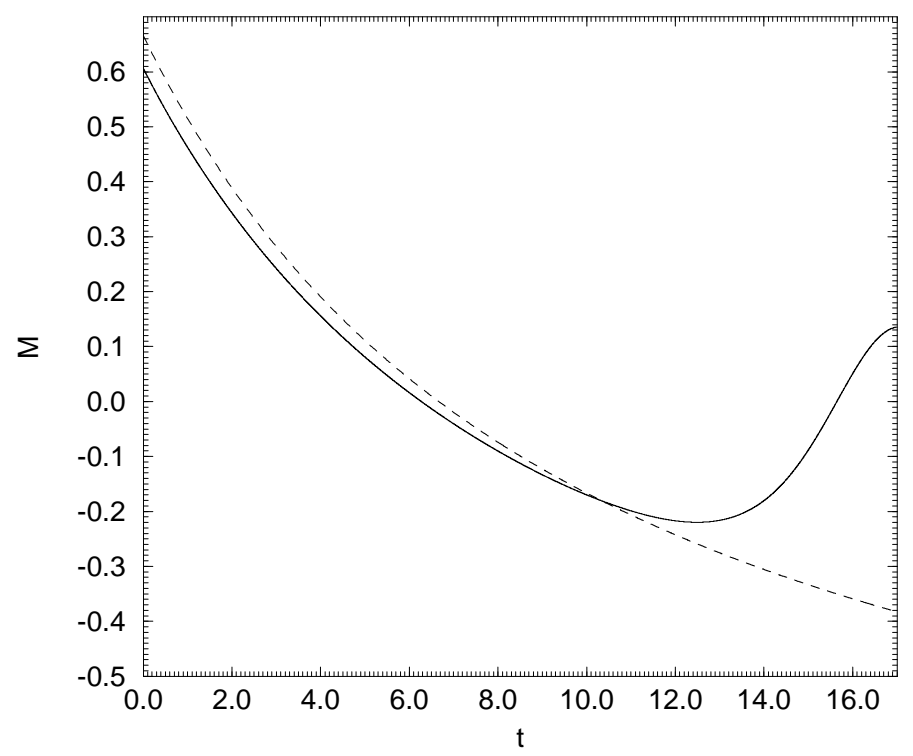

FIG. 8. Plot of the square of the effective mass $M=m_{\text {eff }}^{2}$ vs. cosmological time $t$ in the equilibrium case (dashed line) where $m_{e f f}^{2}=-m^{2}+\frac{\lambda}{24} \frac{T_{0}^{2}}{a^{2}(t)}$ and the in nonequilibrium case (solid line) where $m_{\text {eff }}^{2}$ is determined by the full numerical simulations. The slight difference in the equilibrium and nonequilibrium curves at the beginning of the evolution is due to the difference between the general Hartree-Fock effective mass given by Eq. (2.20) and the high temperature, small $\lambda$ limit given by Eq. (2.21). 